\title{
Exploring the occurrence of thioflavin-T-positive insulin amyloid aggregation intermediates
}

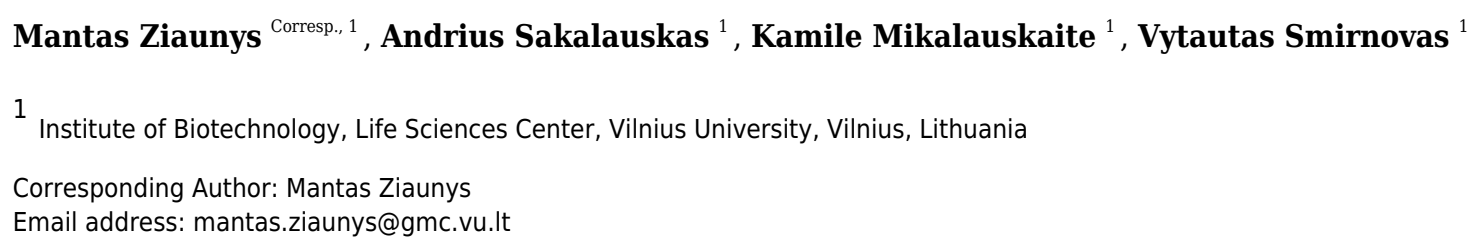

The aggregation of proteins is considered to be the main cause of several neurodegenerative diseases. Despite much progress in amyloid research, the process of fibrillization is still not fully understood, which is one of the main reasons why there are still very few effective treatments available. When the aggregation of insulin, a model amyloidogenic protein, is tracked using thioflavin-T (ThT), an amyloid specific dye, there is an anomalous occurrence of double-sigmoidal aggregation kinetics. Such an event is likely related to the formation of ThT-positive intermediates, which may affect the outcome of both aggregation kinetic data, as well as final fibril structure. In this work we explore insulin fibrillization under conditions, where both normal and double-sigmoidal kinetics are observed and show that, despite their dye-binding properties and random occurrence, the ThT-positive intermediates do not significantly alter the overall aggregation process. 


\section{Exploring the occurrence of thioflavin-T-positive insulin 2 amyloid aggregation intermediates}

3 Mantas Ziaunys*, Andrius Sakalauskas, Kamile Mikalauskaite, Vytautas Smirnovas

4

5 Institute of Biotechnology, Life Sciences Center, Vilnius University, Vilnius, Lithuania

$6 *$ Corresponding Author:

7 Mantas Ziaunys

8 Sauletekio al. 7, Vilnius, LT-10257, Lithuania

9 Email address: mantas.ziaunys@gmc.vu.1t

\section{Abstract}

12 The aggregation of proteins is considered to be the main cause of several neurodegenerative

13 diseases. Despite much progress in amyloid research, the process of fibrillization is still not fully

14 understood, which is one of the main reasons why there are still very few effective treatments available. When the aggregation of insulin, a model amyloidogenic protein, is tracked using thioflavin-T (ThT), an amyloid specific dye, there is an anomalous occurrence of doublesigmoidal aggregation kinetics. Such an event is likely related to the formation of ThT-positive intermediates, which may affect the outcome of both aggregation kinetic data, as well as final fibril structure. In this work we explore insulin fibrillization under conditions, where both normal and double-sigmoidal kinetics are observed and show that, despite their dye-binding properties and random occurrence, the ThT-positive intermediates do not significantly alter the overall 22 aggregation process.

\section{Introduction}

24 Protein aggregation into amyloid fibrils is linked to multiple neurodegenerative disorders, such as Alzheimer's, Parkinson's or prion diseases (Knowles, Vendruscolo \& Dobson, 2014; Chiti \&

26 Dobson, 2017), affecting millions of people worldwide (Isik, 2010). Such protein assembly into 27 insoluble aggregates is still not fully understood, despite the significant effort put into figuring 
out both the mechanism of aggregation (Meisl et al., 2016; Castello et al., 2017; Biza et al., 2017; Giorgetti et al., 2018; Linse, 2019), as well as the resulting fibril structural aspects (Makin \& Serpell, 2005; Fitzpatrick et al., 2013). As a consequence, there are still very few disease modifying drugs available (Mehta et al., 2017; Cummings et al., 2019; Maurer et al., 2018; Park et al., 2020).

The process of amyloid fibril formation consists of multiple microscopic events. The first one being nucleation, a process during which proteins lose their native structure and form a primary aggregation center (Chatani \& Yamamoto, 2018). This structure then passes several growth phases, such as elongation (Gurry \& Stultz, 2014), assembly into protofibrils (Dolui et al., 2018) and subsequent maturation into fully formed fibrils (Ma et al., 2013; Sidhu et al., 2017). The resulting aggregates are then capable of acting as catalysts for surface-mediated secondary nucleation (Törnquist et al., 2018), as well as fragmenting into smaller fibrils (Nicoud et al., 2015), thus creating new aggregation centers. Different proteins have also been shown to form specific oligomeric species prior to further aggregation processes (Nettleton et al., 2000; Chiti \& Dobson, 2006; Danzer et al., 2007; Selivanova \& Galzitskaya, 2012; Sengupta, Nilson \& Kayed, 2016). Such a large number of possible steps involved in the aggregation process significantly complicates matters and requires extensive research in order to understand and prevent the progress of amyloid-related diseases.

In order to study aggregation reactions in vitro, insulin is often used as a model amyloidogenic protein (Brange et al., 1997). Despite its main application as a treatment for diabetes, insulin is capable of forming amyloid fibrils under acidic or neutral $\mathrm{pH}$ at an elevated temperature (Nielsen et al., 2001). This, coupled with its availability, has made it a widely used protein to study both the mechanisms of amyloid formation (Ahmad et al., 2003; Podestà et al., 2006; Malik \& Roy, 2011), as well as possible inhibitory compounds (Wang, Dong \& Sun, 2012; Malisauskas et al., 2015; Zheng \& Lazo, 2018). Even though a large number of experiments have been conducted with insulin under various conditions, new information regarding its fibrillation continues to arise, such as new possible aggregation mechanisms or structural polymorphisms (Sakalauskas, Ziaunys \& Smirnovas, 2019; Ratha et al., 2020). A factor that requires further attention is the seemingly random appearance of double-sigmoidal aggregation kinetics when examining the fibrillization of insulin with a fluorescent probe - thioflavin-T (ThT). This phenomenon was examined by Smirnovas et al. (Smirnovas \& Winter, 2008), Grudzielanek et al. (Grudzielanek, Smirnovas \& Winter, 2006) and Fodera et al. (Foderà et al., 2009), where it was shown that the first increase in the double-sigmoidal curve is likely related to the formation of oligomeric intermediate species capable of binding $\mathrm{ThT}$ and this event was consistently reproducible only under certain environmental conditions.

ThT is a benzothiazole dye that binds to the beta-sheet grooves of amyloid fibrils and attains a locked conformation (Robbins et al., 2012). This causes a red shift of its excitation and emission wavelengths, as well as a significant increase in fluorescence quantum yield (Gade Malmos et al., 2017; Xue et al., 2017). The fluorescence intensity, binding affinity and maximum 
67 excitation/emission wavelengths are highly dependent on the conformation of fibrils and there are even reports of multiple types of binding modes on the same type of aggregate (Sidhu et al., 2018; Ziaunys, Sneideris \& Smirnovas, 2020). Despite being widely used as a probe to track amyloid formation, the dye's fluorescence is not exclusively tied to such fibrillar aggregates, as it has been shown to increase in fluorescence upon binding or being trapped in non-amyloid structures (Singh et al., 2010; Sulatskaya et al., 2018). This, in turn, does not rule out the possibility of the double-sigmoidal kinetics being the result of structures that are not amyloid in nature.

In our research we observed that when human recombinant insulin is aggregated at $\mathrm{pH} 2.4$, there exist both regular sigmoidal fibrilization kinetic curves, as well as double-sigmoidal ones under the same aggregation conditions. Unlike in the previously reported cases (Grudzielanek, Smirnovas \& Winter, 2006; Smirnovas \& Winter, 2008; Foderà et al., 2009), this occurrence appears to be random even when the same batch of protein is used. The double-sigmoidal curves also possess a different ThT fluorescence intensity at the end of the reaction, which begs the question whether the formation of these anomalous ThT-positive intermediates could yield differently structured fibrils. Small variations in insulin aggregation conditions, such as $\mathrm{pH}$ value or protein concentration (Sneideris et al., 2015; Sakalauskas, Ziaunys \& Smirnovas, 2019) can cause the formation of distinct conformation aggregates. Since both regular and doublesigmoidal aggregation types exist under the same conditions, this creates an opportunity to explore any possible differences during the whole fibrillization process. In this work we examine a large sample size of insulin aggregation kinetic curves, isolate the double-sigmoidal kinetic samples from regular ones and determine whether there are secondary structure, morphology, ThT binding and seeding propensity differences between them. In addition, both regular and double-sigmoidal aggregation reactions are tracked by scanning ThT fluorescence excitationemission matrices in order to determine if there are aggregate structural differences from distinct dye binding, such as specific maximum excitation and emission wavelengths or bound-ThT fluorescence intensity (Groenning et al., 2007; Ziaunys \& Smirnovas, 2019b; Ziaunys, Sakalauskas \& Smirnovas, 2020).

\section{Materials and Methods}

\section{Insulin aggregation}

Human recombinant insulin powder (Sigma-Aldrich cat. No. 91077C) was dissolved in a 100 $\mathrm{mM}$ sodium phosphate buffer ( $\mathrm{pH} 2.4$ ) containing $100 \mathrm{mM} \mathrm{NaCl}$ (reaction buffer). ThT (SigmaAldrich cat. No. T3516) was dissolved in $\mathrm{H}_{2} \mathrm{O}$ to a final concentration of $\sim 12 \mathrm{mM}$ and mixed for 10 min using vigorous agitation, after which the dye solution was filtered through a $0.22 \mu \mathrm{m}$ pore syringe filter. An aliquot of the ThT stock solution was diluted 200 times and the exact dye concentration was determined by measuring the solution's absorbance at $412 \mathrm{~nm}\left(\varepsilon_{412}=23250\right.$ $\mathrm{M}^{-1} \mathrm{~cm}^{-1}$ ). The ThT stock solution was then diluted to a final concentration of $10 \mathrm{mM}$. The protein solution was then combined with the reaction buffer and a $10 \mathrm{mM}$ ThTsolution to a final 
105 protein and ThT concentration of $100 \mu \mathrm{M}$ (insulin $\varepsilon_{280}=6335 \mathrm{M}^{-1} \mathrm{~cm}^{-1}, \mathrm{MW}=5808 \mathrm{Da}$ ) and

106 distributed into $200 \mu \mathrm{L}$ test tubes (20 $\mu \mathrm{L}$ final volume). These conditions result in both types of

107 aggregation, with a random appearance of double-sigmoidal kinetic curves.

108 The aggregation kinetics were tracked as previously described (Milto, Michailova \& Smirnovas, 109 2014). In short, sample ThT fluorescence intensity was monitored using a Qiagen Rotorgene Q

110 real-time analyzer at a constant $60^{\circ} \mathrm{C}$ temperature with measurements taken every minute. A total

111 of one thousand samples were measured in batches of 36 . After the aggregation reaction, the

112 samples were stored at $4^{\circ} \mathrm{C}$.

113 For seeded aggregation, fibril samples were sonicated for $10 \mathrm{~min}$ using a Bandelin Sonopuls

114 ultrasonic homogenizer with a MS73 tip (40\% power, with 30 s sonication / 30 s rest intervals).

115 Then insulin, ThT and fibril solutions were combined to a final protein concentration of $100 \mu \mathrm{M}$,

116 ThT concentration of $100 \mu \mathrm{M}$ and $1 \%$ or $10^{-5 \%}$ fibrils ( $\%$ of total protein mass in solution). The

117 reaction was monitored as in the non-seeded aggregation experiment.

\section{Kinetic data analysis}

119 A first-order derivative was calculated for each sample's kinetic data, using a 40-point averaging 120 range. The maximum value of the derivative curve corresponds to the rate of aggregation, while

121 its position - to the time at which the rate is highest $\left(t_{r}\right)$. Samples which had one clear peak in the

122 first-order derivative were regarded as normal, while ones which were composed of two peaks

123 (regular, high-rate peak and a small, low-rate peak preceding it) - as double-sigmoidal. Data

124 processing was done using Origin 2018 software.

\section{Fluorescence measurements}

126 Each sample was diluted 5 times to $100 \mu \mathrm{L}$ with the reaction buffer containing $100 \mu \mathrm{M}$ of ThT

127 and their fluorescence intensity was measured using a Varian Cary Eclipse Fluorescence

128 Spectrophotometer with $440 \mathrm{~nm}$ excitation (slit width $-5 \mathrm{~nm}$ ) and $480 \mathrm{~nm}$ emission (slit width -

$1295 \mathrm{~nm}$ ) wavelengths. For each case, three measurements were taken and averaged.

\section{Atomic force microscopy}

131 The samples were separated into two groups based on their aggregation kinetic profiles and

132 mixed to result in a homogenous solution. $30 \mu \mathrm{L}$ aliquots were deposited on freshly cleaved

133 mica, incubated for $1 \mathrm{~min}$, washed with $1 \mathrm{~mL}$ of MilliQ water and dried under airflow. In the

134 case of intermediate aggregates during the double-sigmoidal aggregation, the real-time analyzer

135 was stopped when the aggregation curve reached the first minor plateau. Then the samples were

136 quickly removed and placed on freshly cleaved mica as mentioned earlier. For each condition,

137 three $10 \times 10 \mu \mathrm{m}$ AFM images were recorded as previously described (Sneideris et al., 2019)

138 using a Dimension Icon (Bruker) atomic force microscope, operating in tapping mode with a

139 silicon cantilever Tap300AI-G (40 N m${ }^{-1}$, Budget Sensors). High resolution (1024 x 1024 pixels)

140 images were flattened and analyzed using Gwyddion 2.5.5 and SPIP 6.7.8. Each fibril's height 
141 was determined by tracing lines perpendicular to the fibril's axis. Height statistical analysis was

142 conducted by taking into consideration all three repeats for each condition, i.e. a similar number

143 of aggregates were examined in every image.

\section{Fourier-transform infrared spectroscopy}

145 The two sample groups were centrifuged at $10^{\prime} 000 \mathrm{~g}$ for $30 \mathrm{~min}$ and resuspended in $1 \mathrm{~mL}$ of $146 \mathrm{D}_{2} \mathrm{O}$. The centrifugation and resuspension step was repeated 3 times and the final resuspension

147 volume was $0.25 \mathrm{~mL}$. Before measurements, both samples were sonicated using a Bandelin

148 Sonopuls ultrasonic homogenizer with a MS72 tip (20\% power and constant sonication for $30 \mathrm{~s}$ ).

149 Sonication helps to break fibril clumps, which leads to less scattering effects and better-quality

150 FTIR spectra. During sample preparation and measurement, H-D exchange is insignificant, as in

151 the case of insulin fibrils it is very slow (Dzwolak, Loksztejn \& Smirnovas, 2006). The spectra

152 were recorded as previously described (Sneideris et al., 2019). In short, the concentrated fibril

153 samples were scanned in near-vacuum conditions ( $\sim 2 \mathrm{mbar})$ at room temperature using a Vertex

$15480 \mathrm{v}$ (Bruker) IR spectrometer. 256 interferograms were averaged for each spectrum. A $\mathrm{D}_{2} \mathrm{O}$

155 spectrum was subtracted and the resulting spectra were normalized to the same area of amide I/I'

156 band (1700-1595 $\left.\mathrm{cm}^{-1}\right)$. Data processing was performed using GRAMS software.

\section{ThT fluorescence excitation-emission matrices}

158 The aggregation solution was prepared as described in the insulin aggregation section to a final

159 volume of $3 \mathrm{~mL}$. The solution was then placed in a $10 \mathrm{~mm}$ pathlength cuvette, sealed with a plug 160 cap to prevent evaporation and incubated at $60^{\circ} \mathrm{C}$ without agitation. EEMs were scanned every 5

161 min using a Varian Cary Eclipse fluorescence spectrophotometer using an excitation range from

162440 to $465 \mathrm{~nm}$ and emission range from 475 to $500 \mathrm{~nm}$ (excitation and emission slit widths -5

$163 \mathrm{~nm}$, wavelength step $-1 \mathrm{~nm}$, scan rate -600 points/min). Due to the analysis being conducted on

164 a transitioning system, the EEM size was optimized to be as minimal as possible to lower the

165 impact of an intensity drift, which results from different concentrations of aggregates present at

166 the start and finish of each scan cycle. This was done by first acquiring a larger EEM, which

167 encompassed the maximum ThT fluorescence zone, then it was narrowed down as much as

168 possible to reduce scan time.

169 Each EEM was corrected for the inner filter effect caused by $100 \mu \mathrm{M}$ of ThT as described

170 previously (Ziaunys \& Smirnovas, 2019b). In short, the correction was made by using the

171 absorbance spectra of $100 \mu \mathrm{M}$ non-bound ThT, as it is extremely difficult to account for

172 absorbance changes throughout the entire reaction resulting from ThT becoming bound to fibrils

173 and because the majority of ThT remains non-bound even when all insulin is aggregated

174 (Ziaunys \& Smirnovas, 2019b). The EEM "center of mass" was then calculated for the entire

175 EEM after the inner filter correction. This was done to prevent signal noise, caused by light

176 scattering, from affecting the maximum intensity position. 


\section{Results}

\section{Aggregation kinetics}

181 A large number ( $\mathrm{n}=1000)$ of low volume insulin samples were aggregated under the exact same 182 conditions and their kinetics were tracked by monitoring changes in ThT fluorescence intensity.

183 Analysis of all the data revealed that a majority of samples experienced normal, sigmoidal 184 spontaneous aggregation kinetics, with one rate maximum seen in the first order derivative

185 (Figure 1A, C). A fraction of samples displayed double-sigmoidal kinetics, with two peaks in the 186 first order derivative curve (Figure 1B, D). The first increase in fluorescence intensity of the 187 double-sigmoidal aggregation kinetics occurred roughly 100 minutes before the second increase 188 and its rate was, on average, nearly 10-fold lower.

189 A total of 55 samples possessed such unusual aggregation kinetics, constituting a probability of 190 such an occurrence being at least $5.5 \%$ under the tested conditions. 77 samples had an 191 intermediate kinetic profile (very small first peak or a large overlap between both peaks), which

192 could not be accurately attributed to either type of aggregation. The normal and double-

193 sigmoidal samples were then separated for further analysis.

194 In order to determine if there are any links between the rate of aggregation, the time at which this 195 rate is highest and the resulting fluorescence intensity of fibrils, all three factor dependencies

196 were examined. We can see that all three parameters are mostly independent from one another 197 (Figure 2A-C). The time at which the aggregation rate is highest, does not influence the rate at 198 which fibril elongation occurs, neither does it change the final fluorescence intensity of the 199 formed fibrils. When we compare these three factors between the normal and double-sigmoidal 200 samples (Figure 2D-I)), it appears that the double-sigmoidal fibrillization has a slightly lower 201 time at which the maximum aggregation rate is reached (Figure 2D, G) and it has no effect on 202 the rate itself (Figure 2E, H). There is, however, a considerable difference in the final

203 fluorescence intensity distribution (Figure 2F, I). The distribution maximum is more than $10 \%$ 204 lower when the aggregation kinetics are double-sigmoidal, suggesting that there are either off205 pathway aggregates or a fraction of fibrils possess a different ThT binding mode. Despite this

206 distinction in average fluorescence intensity, a large portion of all three data sets overlap with

207 one another due to a large spread caused by the stochastic nature of non-seeded insulin

208 aggregation (Foderà et al., 2008).

\section{Fibril structure and seeding properties}

210 The fibril samples were examined using atomic force microscopy (AFM), Fourier-transform

211 infrared spectroscopy (FTIR) and used as seeds to examine their rate of self-replication. In the 
212 AFM images acquired during the first increase in signal intensity during double-sigmoidal

213 kinetics, we observe small, round oligomeric aggregate species (with most having a height of 1

214 to $2 \mathrm{~nm}$ ) and short protofibrils ( 0.1 to $0.5 \mu \mathrm{m}$ in length) (Figure 3A, Figure S1). When compared

215 to a sample obtained before an increase in dye fluorescence is observed (Figure S1), there are far

216 more aggregates present in the case of ThT-positive intermediates. The ThT-negative samples

217 also contain a higher number of $0.5-1.5 \mathrm{~nm}$ height assemblies and very few elongated

218 structures (Figure S2A-C). When the aggregation reactions are concluded, the fibrils are

219 considerably longer and have a greater height, however, there do not seem to be any major

220 differences between both cases, neither visually nor by their height distribution (Figure 3B-D,

221 Figure S1). Due to formation of large aggregate clumps, the fibrils were sonicated to better

222 examine any possible differences in their height. While the height distribution average values are

223 similar, there is a wider spread in the case of the sonicated double-sigmoidal sample (Figure S3),

224 likely caused by the existence of several smaller fibrils or amorphous aggregates. The FTIR

225 second derivative spectra (Figure 3E) and seeding kinetics (Figure 3F) are also nearly identical

226 for both cases, indicating that the double-sigmoidal aggregation does not have a significant

227 influence on the final fibril secondary structure or self-replication properties.

\section{ThT-positive intermediates}

229 The normal and double-sigmoidal aggregation reactions were examined by scanning excitation-

230 emission matrices of ThT fluorescence during aggregation. The kinetic curves were plotted as

231 the maximum EEM signal intensity over time. When examining the kinetics of double-sigmoidal

232 aggregation, we see that the lag phase is followed by a slow increase in ThT fluorescence

233 intensity, then a sudden jump in intensity (marked as *), which then quickly returns to a low

234 value and is continued by the second growth phase (Figure 4A). Such a jump is not visible in the

235 normal aggregation data (Figure 4A), nor in any of the previous experiments, where samples

236 were only scanned once a minute. This indicates that it may only be visible for a very short time

237 during the EEM scan. When the excitation and emission wavelengths of the EEM "center of

238 mass" are calculated (Figure 4B, C), we see that there are significant changes in both of these

239 parameters during the first, anomalous increase in ThT intensity, as compared to relatively minor

240 variations in the normal aggregation. The excitation wavelength shifts from $457 \mathrm{~nm}$ to $453 \mathrm{~nm}$

241 and then rises back to $454 \mathrm{~nm}$, while the emission intensity shifts between $489 \mathrm{~nm}$ and $487 \mathrm{~nm}$

242 and gradually reaches $488 \mathrm{~nm}$. Both wavelengths reach a constant value at roughly the same time

243 after the sudden increase in ThT fluorescence intensity, marking the end of the anomalous phase.

244 If we examine the top fluorescence intensity value distributions in the EEMs before and after the 245 sudden signal jump (Figure 4D), there are both significant shifts in the top value positions during 246 the anomalous phase, as well as single, high intensity lines. Such lines can be caused by either a

247 large particle floating past the optical path during a scan or by sudden association and

248 dissociation of a ThT-positive aggregate. After the high intensity jump, once the signal returns to 
249

250

251

\section{Discussion}

253

254

255

256

257

258

259

260

261

262

263

264

265

266

267

268

269

270

271

272

273

274

275

276

277

278

279

280

281

282

283

284

285

normal, these lines are no longer present in any of the EEMs (as seen after 290 minutes (Figure 4D)) and they become nearly identical. Such an occurrence has been observed multiple times throughout the study and it ranged from being mild (Figure S4) to very extreme (Figure S5).

The large sample size of aggregation reactions shows that under these conditions, the occurrence of such anomalous, double-sigmoidal fibrilization kinetics is both relatively rare $(5.5 \%)$ and seemingly random. This is unlike the previously reported cases, where a certain set of conditions caused all of the kinetic curves to be double-sigmoidal (Grudzielanek, Smirnovas \& Winter, 2006; Smirnovas \& Winter, 2008; Foderà et al., 2009). The conditions used in this work allowed to examine how such peculiar kinetics affected the overall aggregation reaction, as both types of fibrillizations were observed. When compared to normal aggregation, this anomalous event occurs roughly 100 minutes before the second increase, however it does not influence the time at which maximum aggregation rate is reached nor the rate of aggregation and the resulting fibrils have an identical morphology, secondary structure and seeding properties. It is possible that it generates a small population of small, amorphous structures, as hinted by the fibril height range, AFM images and the lower ThT fluorescence intensity distribution. The fact that this anomaly does not influence the overall kinetic parameters (apart from lag time) or fibril structure is a positive aspect, considering that these values are often used to determine the effectiveness of anti-amyloid compounds. However, the random formation of different intermediate species does raise concerns. If these structures can appear in any reaction solution and there is minimal connection between their existence and the overall aggregation process, then there is essentially no way of controlling this event.

Judging from the ThT fluorescence EEMs, it appears that during the anomalous aggregation phase, there exists the formation of ThT-positive intermediates or structures that are capable of trapping and conformationally "locking" the dye molecules, unlike during a normal fibrilization process. AFM images acquired during this phase show small and round aggregates, as well as short protofibril species, with an average height that is much lower than observed for fully formed fibrils. Their ability to bind ThT in a different mode (as identified by the higher excitation and emission wavelengths) suggests that they possess a structure that is distinct from both normal intermediate species, as well as the fully formed fibrils. The differences in these parameters could also be an indicator that there is no actual surface-dye interaction, but rather an entrapment of ThT in the oligomeric structure. The lower ThT fluorescence intensity distribution at the end of the reaction and AFM images also hint at a possibility that some of these structures remain in solution and do not become incorporated into the amyloid structure of normal fibrils.

A possible explanation for the ThT-binding ability of these intermediates may stem from the high intensity lines seen in the ThT fluorescence EEMs. When the sample is continuously scanned to generate an EEM, such lines can only result from either a larger particle floating past 
286 the optical path or by a quick association and dissociation of a ThT positive aggregate.

287 Considering that during fibrilization the concentration of large aggregates increases, an event

288 which results in enhanced light scattering and signal noise, we would expect to observe an

289 increasing amount of such high intensity lines, however, this is not the case. Once the first phase

290 of the double-sigmoidal kinetics is concluded, they are no longer seen, which means that they are

291 likely not the result of light scattering from larger aggregate particles. This leaves the hypothesis

292 that ThT-positive intermediates quickly associate and dissociate during this time period. If these

293 intermediates are capable of trapping ThT molecules within their structure, they may not even

294 require a similarity to amyloids, as it has been shown that ThT immobilization can cause an

295 increase in fluorescence intensity (Hutter et al., 2011; Ziaunys \& Smirnovas, 2019a). This would

296 also explain the significantly different excitation and emission wavelengths, as these parameters

297 depend highly on the dye's binding mode.

298 While these ThT-positive intermediates do not have any major effect on the final fibril structure

299 and most kinetic parameters, they could become an issue when testing potential anti-amyloid

300 compounds. If an inhibitor targets the process of primary nucleation and is specific towards a

301 certain type of intermediate structure, the formation of a different type of aggregate, which

302 incorporates ThT but is not amyloid-like, may not be affected at all and result in an increase in

303 ThT fluorescence intensity. This would then lead to a false interpretation on the effectiveness of

304 the tested compound and negatively affect the drug screening process.

305

306 Conclusions

307 The occurrence of double-sigmoidal kinetics during insulin amyloid aggregation does not

308 influence the final fibril structure or morphology, nor does it change the rate of the main

309 reaction. However, it does result in a lower ThT fluorescence intensity and may be related to the

310 formation of a different type of aggregates. The random variability observed during intermediate

311 oligomer formation may also have a negative impact during potential anti-amyloid drug

312 screenings and lead to false interpretations. 


\section{References}

314

315

316

317

318

319

320

321

322

323

324

325

326

327

328

329

330

331

332

333

334

335

336

337

338

339

340

341

342

343

344

345

346

347

348

Ahmad A, Millett IS, Doniach S, Uversky VN, Fink AL. 2003. Partially Folded Intermediates in Insulin Fibrillation. Biochemistry 42:11404-11416. DOI: 10.1021/bi034868o.

Biza K V., Nastou KC, Tsiolaki PL, Mastrokalou C V., Hamodrakas SJ, Iconomidou VA. 2017. The amyloid interactome: Exploring protein aggregation. PLOS ONE 12:e173163. DOI: 10.1371/journal.pone. 0173163 .

Brange J, Andersen L, Laursen ED, Meyn G, Rasmussen E. 1997. Toward Understanding Insulin Fibrillation. Journal of Pharmaceutical Sciences 86:517-525. DOI: 10.1021/js960297s.

Castello F, Paredes JM, Ruedas-Rama MJ, Martin M, Roldan M, Casares S, Orte A. 2017. TwoStep Amyloid Aggregation: Sequential Lag Phase Intermediates. Scientific Reports 7:40065. DOI: $10.1038 /$ srep40065.

Chatani E, Yamamoto N. 2018. Recent progress on understanding the mechanisms of amyloid nucleation. Biophysical Reviews 10:527-534. DOI: 10.1007/s12551-017-0353-8.

Chiti F, Dobson CM. 2006. Protein Misfolding, Functional Amyloid, and Human Disease. Annual Review of Biochemistry 75:333-366. DOI: 10.1146/annurev.biochem.75.101304.123901.

Chiti F, Dobson CM. 2017. Protein Misfolding, Amyloid Formation, and Human Disease: A Summary of Progress Over the Last Decade. Annual Review of Biochemistry 86:27-68. DOI: 10.1146/annurev-biochem- 061516-045115.

Cummings J, Lee G, Ritter A, Sabbagh M, Zhong K. 2019. Alzheimer's disease drug development pipeline: 2019. Alzheimer's \& Dementia: Translational Research \& Clinical Interventions 5:272-293. DOI: $10.1016 /$ j.trci.2019.05.008.

Danzer KM, Haasen D, Karow AR, Moussaud S, Habeck M, Giese A, Kretzschmar H, Hengerer B, Kostka M. 2007. Different Species of $\alpha$-Synuclein Oligomers Induce Calcium Influx and Seeding. Journal of Neuroscience 27:9220-9232. DOI: 10.1523/JNEUROSCI.261707.2007.

Dolui S, Roy A, Pal U, Saha A, Maiti NC. 2018. Structural Insight of Amyloidogenic Intermediates of Human Insulin. ACS Omega 3:2452-2462. DOI: 10.1021/acsomega. $7 \mathrm{~b} 01776$.

Dzwolak W, Loksztejn A, Smirnovas V. 2006. New Insights into the Self-Assembly of Insulin Amyloid Fibrils: An H-D Exchange FT-IR Study. Biochemistry 45:8143-8151. DOI: 10.1021/bi060341a.

Fitzpatrick AWP, Debelouchina GT, Bayro MJ, Clare DK, Caporini MA, Bajaj VS, Jaroniec CP, Wang L, Ladizhansky V, Muller SA, MacPhee CE, Waudby CA, Mott HR, De Simone A, Knowles TPJ, Saibil HR, Vendruscolo M, Orlova E V., Griffin RG, Dobson CM. 2013. Atomic structure and hierarchical assembly of a cross- $\beta$ amyloid fibril. Proceedings of the National Academy of Sciences 110:5468-5473. DOI: 10.1073/pnas.1219476110.

Foderà V, Cataldo S, Librizzi F, Pignataro B, Spiccia P, Leone M. 2009. Self-Organization 
349

350

351

352

353

354

355

356

357

358

359

360

361

362

363

364

365

366

367

368

369

370

371

372

373

374

375

376

377

378

379

380

381

382

383

384

Pathways and Spatial Heterogeneity in Insulin Amyloid Fibril Formation. The Journal of Physical Chemistry B 113:10830-10837. DOI: 10.1021/jp810972y.

Foderà V, Librizzi F, Groenning M, Van De Weert M, Leone M. 2008. Secondary nucleation and accessible surface in insulin amyloid fibril formation. Journal of Physical Chemistry B 112:3853-3858. DOI: $10.1021 / \mathrm{jp} 710131 \mathrm{u}$.

Gade Malmos K, Blancas-Mejia LM, Weber B, Buchner J, Ramirez-Alvarado M, Naiki H, Otzen D. 2017. ThT 101: a primer on the use of thioflavin $\mathrm{T}$ to investigate amyloid formation. Amyloid 24:1-16. DOI: 10.1080/13506129.2017.1304905.

Giorgetti S, Greco C, Tortora P, Aprile F. 2018. Targeting Amyloid Aggregation: An Overview of Strategies and Mechanisms. International Journal of Molecular Sciences 19:2677. DOI: 10.3390/ijms 19092677.

Groenning M, Norrman M, Flink JM, van de Weert M, Bukrinsky JT, Schluckebier G, Frokjaer S. 2007. Binding mode of Thioflavin T in insulin amyloid fibrils. Journal of Structural Biology 159:483-497. DOI: 10.1016/j.jsb.2007.06.004.

Grudzielanek S, Smirnovas V, Winter R. 2006. Solvation-assisted Pressure Tuning of Insulin Fibrillation: From Novel Aggregation Pathways to Biotechnological Applications. Journal of Molecular Biology 356:497-509. DOI: 10.1016/j.jmb.2005.11.075.

Gurry T, Stultz CM. 2014. Mechanism of Amyloid- $\beta$ Fibril Elongation. Biochemistry 53:69816991. DOI: 10.1021/bi500695g.

Hutter T, Amdursky N, Gepshtein R, Elliott SR, Huppert D. 2011. Study of Thioflavin-T Immobilized in Porous Silicon and the Effect of Different Organic Vapors on the Fluorescence Lifetime. Langmuir 27:7587-7594. DOI: 10.1021/la200875k.

Isik AT. 2010. Late onset Alzheimer's disease in older people. Clinical Interventions in Aging 5:307. DOI: 10.2147/CIA.S11718.

Knowles TPJ, Vendruscolo M, Dobson CM. 2014. The amyloid state and its association with protein misfolding diseases. Nature Reviews Molecular Cell Biology 15:384-396. DOI: $10.1038 / \mathrm{nrm} 3810$.

Linse S. 2019. Mechanism of amyloid protein aggregation and the role of inhibitors. Pure and Applied Chemistry 91:211-229. DOI: 10.1515/pac-2018-1017.

Ma J, Komatsu H, Kim YS, Liu L, Hochstrasser RM, Axelsen PH. 2013. Intrinsic Structural Heterogeneity and Long-Term Maturation of Amyloid $\beta$ Peptide Fibrils. ACS Chemical Neuroscience 4:1236-1243. DOI: 10.1021/cn400092v.

Makin OS, Serpell LC. 2005. Structures for amyloid fibrils. FEBS Journal 272:5950-5961. DOI: 10.1111/j.1742-4658.2005.05025.x.

Malik R, Roy I. 2011. Probing the mechanism of insulin aggregation during agitation. International Journal of Pharmaceutics 413:73-80. DOI: 10.1016/j.jpharm.2011.04.024. 
385

386

387

388

389

390

391

392

393

394

395

396

397

398

399

400

401

402

403

404

405

406

407

408

409

410

411

412

413

414

415

416

417

418

419

420

421

422

Malisauskas R, Botyriute A, Cannon JG, Smirnovas V. 2015. Flavone Derivatives as Inhibitors of Insulin Amyloid-Like Fibril Formation. PLOS ONE 10:e0121231. DOI: 10.1371/journal.pone.0121231.

Maurer MS, Schwartz JH, Gundapaneni B, Elliott PM, Merlini G, Waddington-Cruz M, Kristen A V., Grogan M, Witteles R, Damy T, Drachman BM, Shah SJ, Hanna M, Judge DP, Barsdorf AI, Huber P, Patterson TA, Riley S, Schumacher J, Stewart M, Sultan MB, Rapezzi C. 2018. Tafamidis Treatment for Patients with Transthyretin Amyloid Cardiomyopathy. New England Journal of Medicine 379:1007-1016. DOI: 10.1056/NEJMoa1805689.

Mehta D, Jackson R, Paul G, Shi J, Sabbagh M. 2017. Why do trials for Alzheimer's disease drugs keep failing? A discontinued drug perspective for 2010-2015. Expert Opinion on Investigational Drugs 26:735-739. DOI: 10.1080/13543784.2017.1323868.

Meisl G, Kirkegaard JB, Arosio P, Michaels TCT, Vendruscolo M, Dobson CM, Linse S, Knowles TPJ. 2016. Molecular mechanisms of protein aggregation from global fitting of kinetic models. Nature Protocols 11:252-272. DOI: 10.1038/nprot.2016.010.

Milto K, Michailova K, Smirnovas V. 2014. Elongation of Mouse Prion Protein Amyloid-Like Fibrils: Effect of Temperature and Denaturant Concentration. PLoS ONE 9:e94469. DOI: 10.1371/journal.pone.0094469.

Nettleton EJ, Tito P, Sunde M, Bouchard M, Dobson CM, Robinson C V. 2000. Characterization of the Oligomeric States of Insulin in Self-Assembly and Amyloid Fibril Formation by Mass Spectrometry. Biophysical Journal 79:1053-1065. DOI: 10.1016/S0006-3495(00)76359-4.

Nicoud L, Lazzari S, Balderas Barragán D, Morbidelli M. 2015. Fragmentation of Amyloid Fibrils Occurs in Preferential Positions Depending on the Environmental Conditions. The Journal of Physical Chemistry B 119:4644-4652. DOI: 10.1021/acs.jpcb.5b01160.

Nielsen L, Khurana R, Coats A, Frokjaer S, Brange J, Vyas S, Uversky VN, Fink AL. 2001. Effect of environmental factors on the kinetics of insulin fibril formation: Elucidation of the molecular mechanism. Biochemistry 40:6036-6046. DOI: 10.1021/bi002555c.

Park J, Egolum U, Parker S, Andrews E, Ombengi D, Ling H. 2020. Tafamidis: A First-in-Class Transthyretin Stabilizer for Transthyretin Amyloid Cardiomyopathy. Annals of Pharmacotherapy 54:470-477. DOI: 10.1177/1060028019888489.

Podestà A, Tiana G, Milani P, Manno M. 2006. Early Events in Insulin Fibrillization Studied by Time-Lapse Atomic Force Microscopy. Biophysical Journal 90:589-597. DOI: 10.1529/biophysj.105.068833.

Ratha BN, Kar RK, Bednarikova Z, Gazova Z, Kotler SA, Raha S, De S, Maiti NC, Bhunia A. 2020. Molecular Details of a Salt Bridge and Its Role in Insulin Fibrillation by NMR and Raman Spectroscopic Analysis. The Journal of Physical Chemistry B 124:1125-1136. DOI: 10.1021/acs.jpcb.9b10349.

Robbins KJ, Liu G, Selmani V, Lazo ND. 2012. Conformational analysis of thioflavin T bound to the surface of amyloid fibrils. Langmuir 28:16490-16495. DOI: 10.1021/1a303677t.

Peer) reviewing PDF | (2020:10:54921:1:0:NEW 13 Jan 2021) 
423

424

425

426

427

428

429

430

431

432

433

434

435

436

437

438

439

440

441

442

443

444

445

446

447

448

449

450

451

452

453

454

455

456

457

458

459

460

Sakalauskas A, Ziaunys M, Smirnovas V. 2019. Concentration-dependent polymorphism of insulin amyloid fibrils. PeerJ 7:e8208. DOI: 10.7717/peerj.8208.

Selivanova OM, Galzitskaya O V. 2012. Structural polymorphism and possible pathways of amyloid fibril formation on the example of insulin protein. Biochemistry (Moscow) 77:12371247. DOI: $10.1134 / \mathrm{S} 0006297912110028$.

Sengupta U, Nilson AN, Kayed R. 2016. The Role of Amyloid- $\beta$ Oligomers in Toxicity, Propagation, and Immunotherapy. EBioMedicine 6:42-49. DOI: 10.1016/j.ebiom.2016.03.035.

Sidhu A, Segers-Nolten I, Raussens V, Claessens MMAE, Subramaniam V. 2017. Distinct Mechanisms Determine $\alpha$-Synuclein Fibril Morphology during Growth and Maturation. ACS Chemical Neuroscience 8:538-547. DOI: 10.1021/acschemneuro.6b00287.

Sidhu A, Vaneyck J, Blum C, Segers-Nolten I, Subramaniam V. 2018. Polymorph-specific distribution of binding sites determines thioflavin- $T$ fluorescence intensity in $\alpha$-synuclein fibrils. Amyloid 25:189-196. DOI: 10.1080/13506129.2018.1517736.

Singh PK, Kumbhakar M, Pal H, Nath S. 2010. Viscosity Effect on the Ultrafast Bond Twisting Dynamics in an Amyloid Fibril Sensor: Thioflavin-T. The Journal of Physical Chemistry B 114:5920-5927. DOI: 10.1021/jp100371s.

Smirnovas V, Winter R. 2008. Revealing Different Aggregation Pathways of Amyloidogenic Proteins by Ultrasound Velocimetry. Biophysical Journal 94:3241-3246. DOI: 10.1529/biophysj.107.123133.

Sneideris T, Darguzis D, Botyriute A, Grigaliunas M, Winter R, Smirnovas V. 2015. pH-Driven Polymorphism of Insulin Amyloid-Like Fibrils. PLOS ONE 10:e0136602. DOI: 10.1371/journal.pone.0136602.

Sneideris T, Sakalauskas A, Sternke-Hoffmann R, Peduzzo A, Ziaunys M, Buell AK, Smirnovas V. 2019. The Environment Is a Key Factor in Determining the Anti-Amyloid Efficacy of EGCG. Biomolecules 9:1-17. DOI: 10.3390/biom9120855.

Sulatskaya AI, Rychkov GN, Sulatsky MI, Rodina NP, Kuznetsova IM, Turoverov KK. 2018. Thioflavin $T$ Interaction with Acetylcholinesterase: New Evidence of 1:1 Binding Stoichiometry Obtained with Samples Prepared by Equilibrium Microdialysis. ACS Chemical Neuroscience 9:1793-1801. DOI: 10.1021/acschemneuro.8b00111.

Törnquist M, Michaels TCT, Sanagavarapu K, Yang X, Meisl G, Cohen SIA, Knowles TPJ, Linse S. 2018. Secondary nucleation in amyloid formation. Chemical Communications 54:86678684. DOI: $10.1039 / \mathrm{C} 8 \mathrm{CC} 02204 \mathrm{~F}$.

Wang S-H, Dong X-Y, Sun Y. 2012. Effect of (-)-epigallocatechin-3-gallate on human insulin fibrillation/aggregation kinetics. Biochemical Engineering Journal 63:38-49. DOI: 10.1016/j.bej.2012.02.002.

Xue C, Lin TY, Chang D, Guo Z. 2017. Thioflavin T as an amyloid dye: fibril quantification, optimal concentration and effect on aggregation. Royal Society Open Science 4:160696. DOI:

Peer] reviewing PDF | (2020:10:54921:1:0:NEW 13 Jan 2021) 
Zheng Q, Lazo ND. 2018. Mechanistic Studies of the Inhibition of Insulin Fibril Formation by

Ziaunys M, Smirnovas V. 2019a. Emergence of visible light optical properties of L-phenylalanine 466 aggregates. PeerJ 7:e6518. DOI: 10.7717/peerj.6518.

467 Ziaunys M, Smirnovas V. 2019b. Additional Thioflavin-T Binding Mode in Insulin Fibril Inner 468 Core Region. The Journal of Physical Chemistry B 123:8727-8732. DOI: $469 \quad$ 10.1021/acs.jpcb.9b08652.

470 Ziaunys M, Sakalauskas A, Smirnovas V. 2020. Identifying Insulin Fibril Conformational 471 Differences by Thioflavin-T Binding Characteristics. Biomacromolecules 21:4989-4997. 472 DOI: $10.1021 /$ acs.biomac.0c01178.

473 Ziaunys M, Sneideris T, Smirnovas V. 2020. Formation of distinct prion protein amyloid fibrils 474 475 under identical experimental conditions. Scientific Reports 10:4572. DOI: 10.1038/s41598020-61663-2. 
Figure 1

Insulin aggregation curves and their derivatives.

Normal, sigmoidal (A) and anomalous, double-sigmoidal (B) insulin aggregation kinetics and their first order derivatives ( $C, D$ respectively).
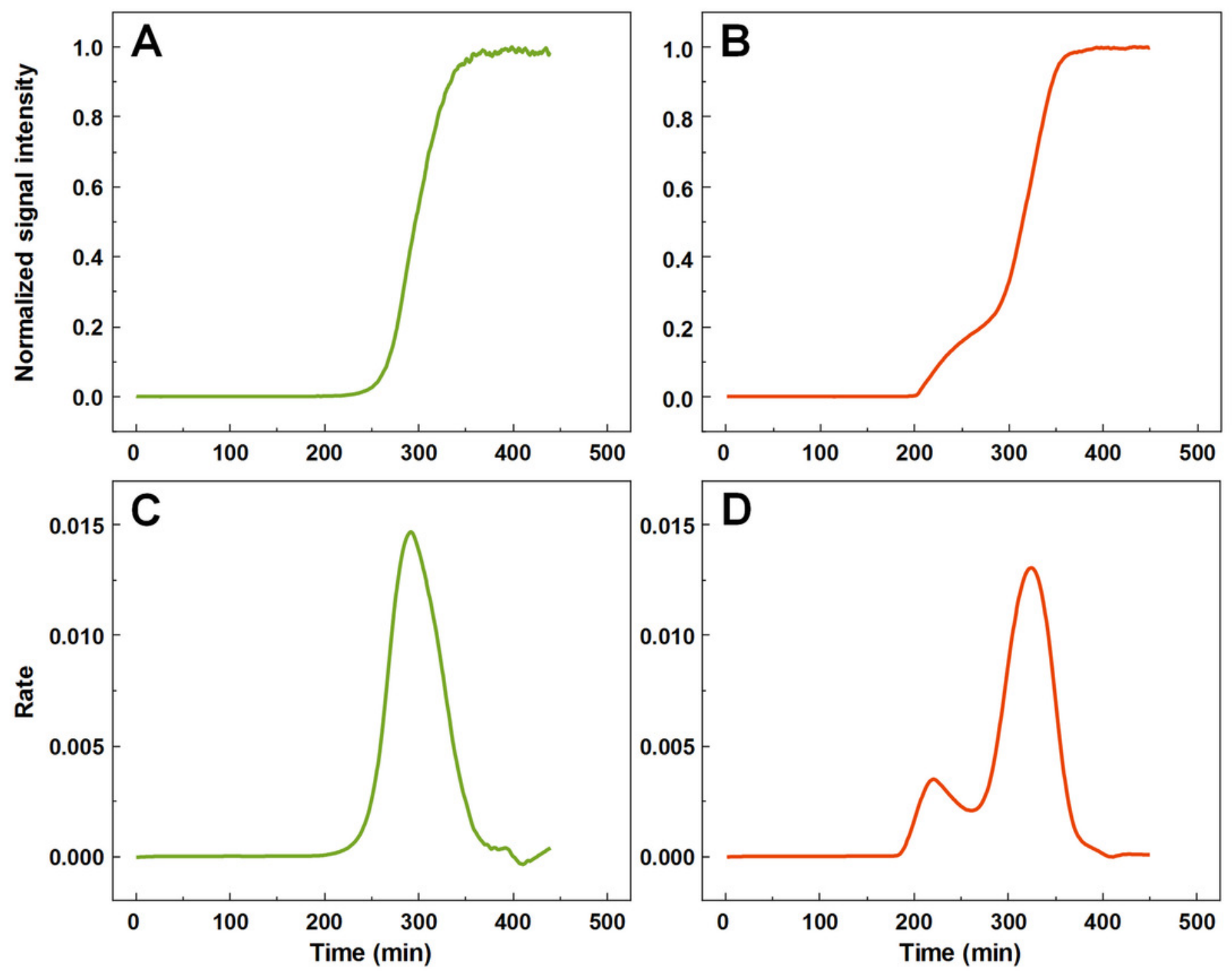


\section{Figure 2}

Distribution of maximum insulin aggregation rate, the time at which it is reached $\left(\mathrm{t}_{\mathrm{r}}\right)$ and final fibril fluorescence intensity.

Dependence of aggregation rate on $t_{r}(A)$, fluorescence intensity on $t_{r}(B)$ and aggregation rate on fluorescence intensity $(C)$. Distribution of $t_{r}(D, G)$, aggregation rate $(E, H)$ and fluorescence intensity $(F, I)$ for normal and double-sigmoidal samples respectively. Colorcoded numbers, displayed above peak-fit curves, indicate peak maximum values. 

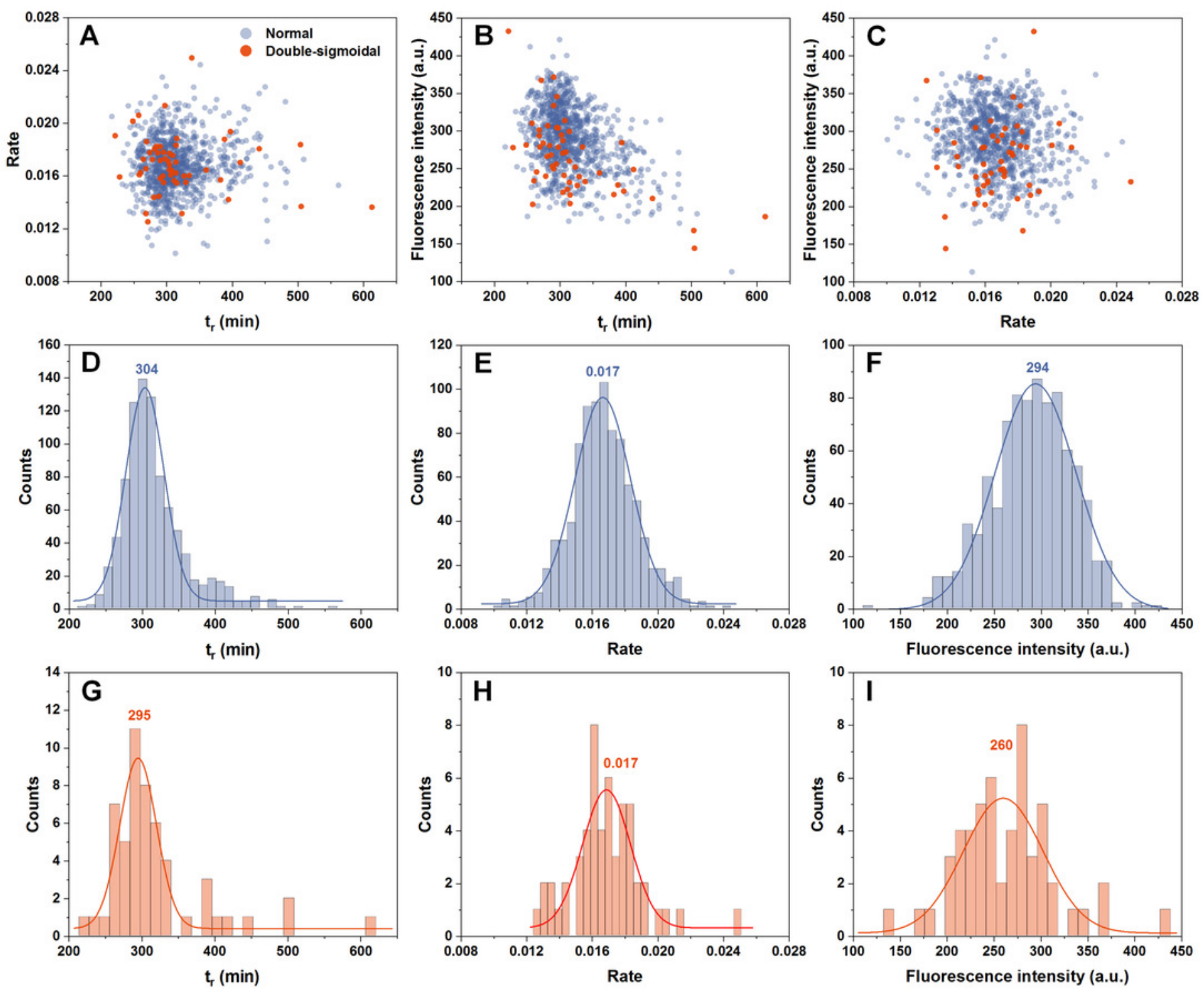


\section{Figure 3}

Normal, intermediate and double-sigmoidal sample AFM images, fibril height distributions, second order FTIR spectra and seeding kinetics.

AFM images of insulin aggregates during the first part of the double-sigmoidal kinetics (A) and at the end of normal (B) and double-sigmoidal (C) aggregation. Height distribution of double-sigmoidal aggregation intermediates and fibrils after normal and double-sigmoidal aggregation (D), where box plots indicate the interquartile range and error bars are one standard deviation. Second order FTIR spectra (E) and seeded aggregation kinetics (F).
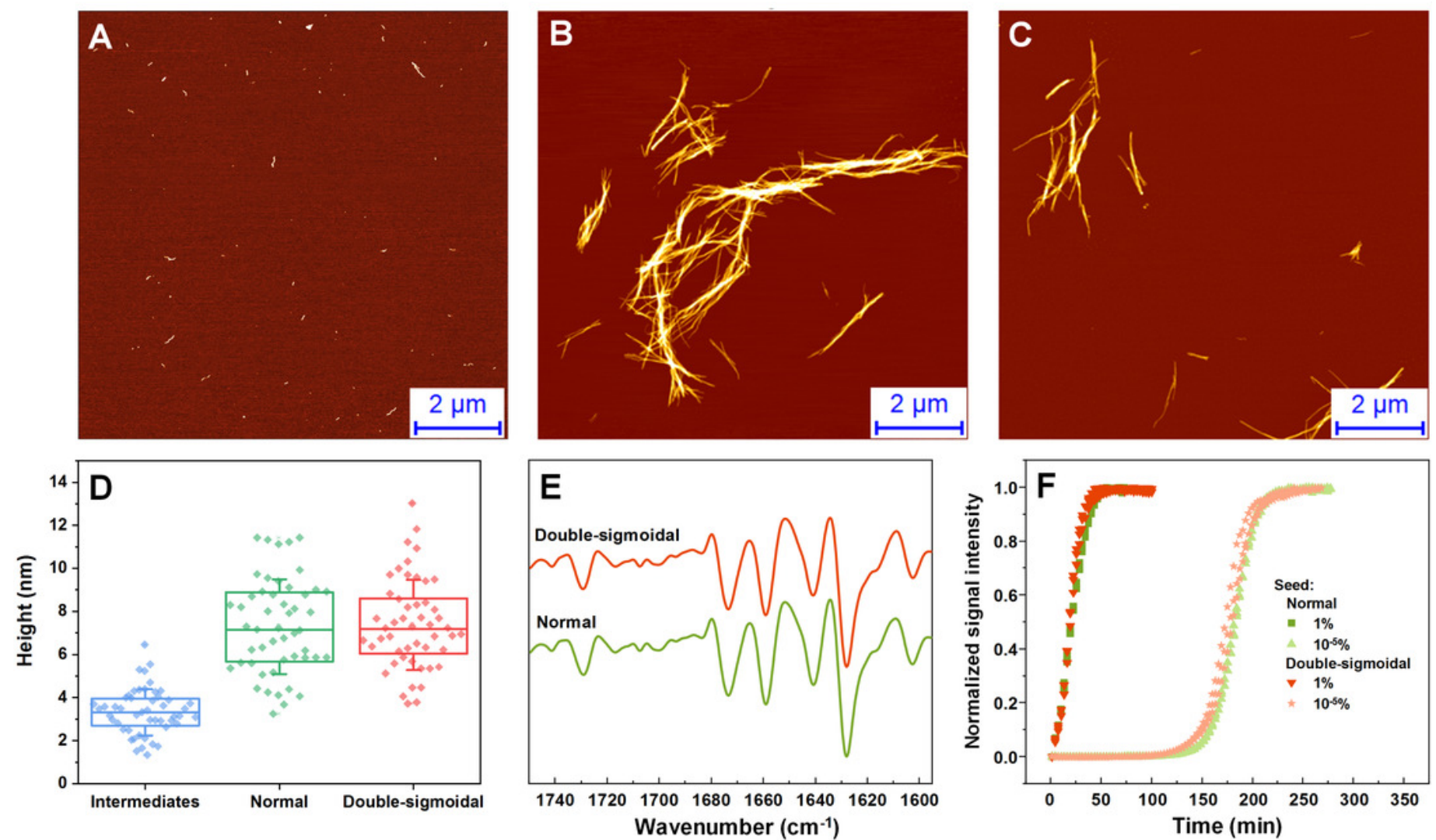


\section{Figure 4}

Insulin aggregation kinetics and bound ThT fluorescence EEMs.

Insulin aggregation kinetics monitored by scanning ThT fluorescence EEMs (A), where each data point is the maximum value in the recorded EEM. EEM "center of mass" excitation (B) and emission (C) wavelengths over the course of aggregation. Top intensity values present in the ThT EEMs at different aggregation time points (D) during the first double-sigmoidal increase (darker green areas represent higher intensity zones). The red $*$ symbol indicates the point where there is a sudden jump in ThT fluorescence intensity. Data in part (A) was fit using a Boltzmann's sigmoidal equation with the anomalous aggregation phase data points omitted from the fitting procedure.
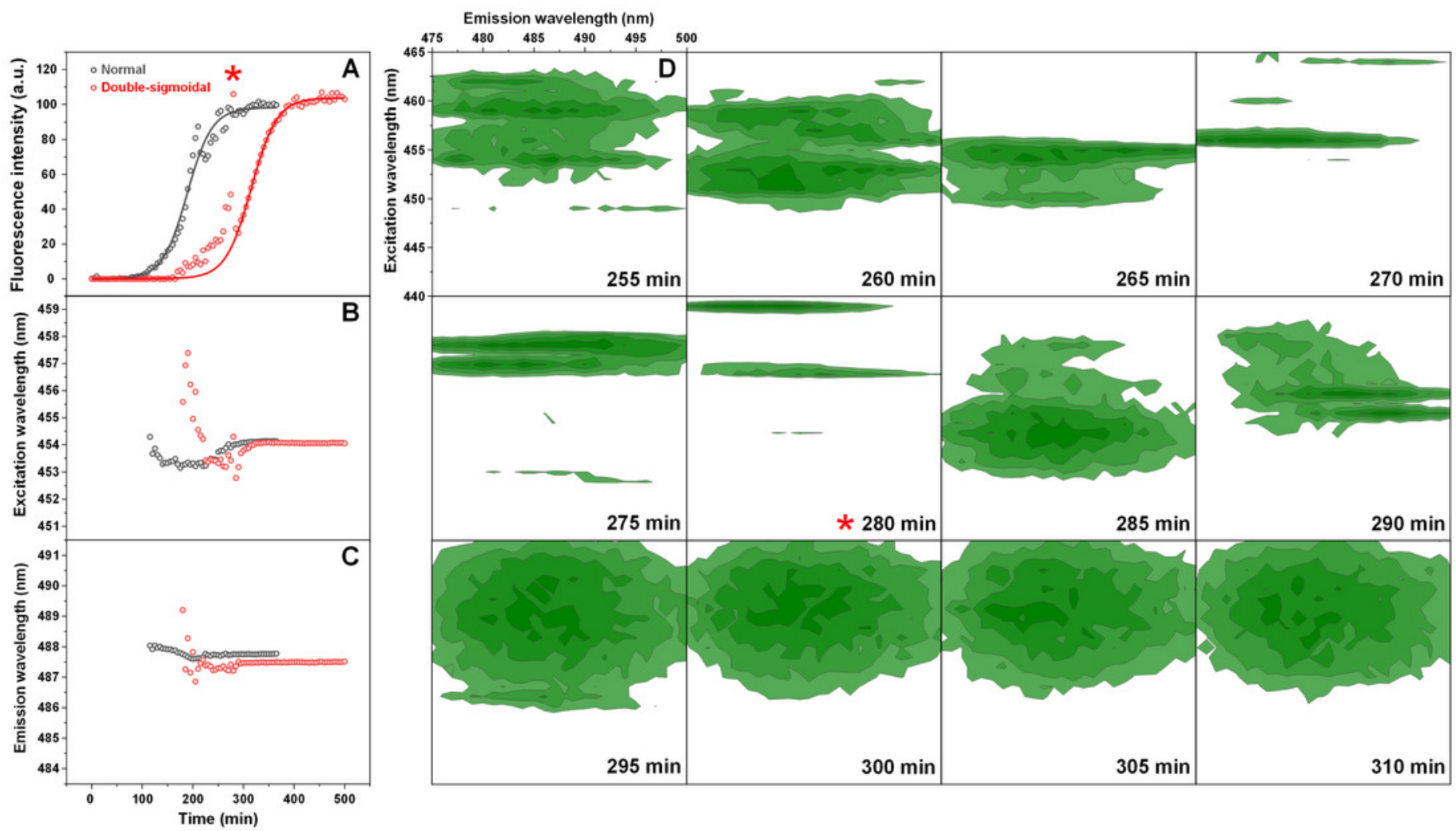